\title{
Modeling and Evaluating Targeted Attacks in Large Scale Dynamic Systems
}

\author{
Emmanuelle Anceaume \\ CNRS IRISA, France \\ anceaume@irisa.fr
}

\author{
Bruno Sericola \\ INRIA Rennes Bretagne-Atlantique, France \\ sericola@inria.fr
}

\author{
Romaric Ludinard, Frédéric Tronel \\ Supélec, France \\ frederic.tronel@rennes.supelec.fr \\ romaric.ludinard@rennes.supelec.fr
}

\begin{abstract}
In this paper we consider the problem of targeted attacks in large scale peer-to-peer overlays. These attacks aimed at exhausting key resources of targeted hosts to diminish their capacity to provide or receive services. To defend the system against such attacks, we rely on clustering and implement induced churn to preserve randomness of nodes identifiers so that adversarial predictions are impossible. We propose robust join, leave, merge and split operations to discourage brute force denial of services and pollution attacks. We show that combining a small amount of randomization in the operations, and adequately tuning the sojourn time of peers in the same region of the overlay allows first to decrease the effect of targeted attacks at cluster level, and second to prevent pollution propagation in the whole overlay.
\end{abstract}

Keywords-Clusterized P2P Overlays, Adversary, Churn, Collusion, Markov chains.

\section{INTRODUCTION}

In this paper we consider the problem of targeted attacks in large scale peer-to-peer systems. These attacks aimed at exhausting key resources of targeted hosts to diminish their capacity to provide or receive services [1], at preventing data indexed at targeted nodes from being discovered and retrieved by poisoning their routing tables, or simply at rerouting or dropping messages addressed to targeted nodes. Such malicious behaviors have led to the proposition of malicious-resilient overlay systems (e.g., [2], [3], [4]). In all these systems, it is assumed that at any time, and anywhere in the overlay, the proportion of compromised peers is bounded and known. Unfortunately, targeted attacks violate such an assumption. It has been shown that peer-to-peer overlays can survive these attacks only if malicious nodes are not able to isolate honest nodes within the system. This is achieved by i) preserving randomness of nodes identifiers [5], and ii) limiting the period of time where nodes can stay at the same position in the overlay. Induced churn has been shown to be a fundamental ingredient to preserve randomness. Churn is classically defined as the rate of turnover of peers in the system [6], and thus induced churn refers to the general idea of forcing peers to move within the system. Several strategies based on this principle have been proposed. Most of them are based on locally induced churn. However either they were proven incorrect or they involve a too high level of complexity to be practically acceptable [5]. Some other strategies, based on globally induced churn, force each node to periodically leave and re-join the system. This may be enforced through nodes limited lifetime in the system. If not properly handled, these solutions keep the system in an unnecessary hyper-activity, which increases accordingly the impact of churn.

In the present work, we investigate adversarial strategies that aim at isolating honest nodes in cluster based overlays, and we present an analytical study of the long term behavior of the system. Our analysis shows that $i$ ) by limiting the sojourn time of nodes at the same position in the overlay and ii) by introducing randomness in the operations of the overlay, pollution attacks are severely reduced at cluster level and do not propagate to the whole overlay. A preliminary work [7] investigates adversarial strategies in the specific context where the sequence of join and leave events is well interleaved. In this paper we extend this preliminary work to a general context in which clusters size varies with churn and thus, undergo merge and split operations. To the best of our knowledge, this is the first work that has conducted such a study.

The remainder of this paper is as follows: In Section II, we present existing works that focus on making structuredbased overlays robust against attacks. In Section III we briefly describe the main features of cluster-based overlays, and present the assumptions made in this work. Section IV describes the robust operations implemented in the overlay. Then, in Section V, we specify the strategy the adversary adopts to perform targeted attacks in the system. The adversarial behavior is modeled and its impact at both cluster level and at the overlay level are respectively studied in Sections VI and VIII. Section IX concludes.

\section{RELATED WORK}

Different approaches have been proposed to face adversarial setting, each one focusing on a particular adversary strategy. Regarding eclipse attacks, a very common technique, called constrained routing table, consists in selecting neighbors based on their identifiers so that all of them are close to some particular points in the identifier space [8]. Such an approach has been implemented into several overlays (e.g., [9], [10], [11]). Another defense against those attacks comes from the observation that during eclipse attacks, the degree of attackers is much higher than the average degree of peers in the overlay. Addressing such attacks consists in bounding peers degrees. Singh et al. [12] propose to 
anonymously audit peers to continuously check the bounded degree of peers. Results of their experimentation show that their solution is effective in an overlay with low to moderately high churn. More generally, the seminal works on DHT routing security by Castro et al. [8] and Sit and Morris [13] combine routing failure tests and redundant routing as a solution to ensure robust routing. Their approach has then been successfully implemented in different structured-based overlays (e.g., [2], [4], [14]). In all these above cited works, it is assumed that at any time, and anywhere in the overlay, the proportion of compromised peers is bounded and $a$ priori known. It allows powerful building blocks such as Byzantine tolerant agreement protocols to be used among peers subsets [2], [4]. When such an assumption does not hold, additional mechanisms are needed. Awerbuch et al [5] propose the Cuckoo\&flip strategy. This strategy consists in introducing local induced churn upon each join and leave operation. This strategy prevents malicious peers from predicting what is going to be the state of the overlay after a given sequence of join and leave operations. Subsequently to this work, experiments have been conducted to check the feasibility of global induced churn. These experiments assume that the overlay is populated by no more than $25 \%$ of compromised peers [15], or that the topology of the overlay is static [7].

\section{SySTEM MODEL}

\section{A. Model of the Network.}

We consider a dynamic system populated by a large collection of peers in which each peer is assigned a unique and permanent random identifier from an $m$-bit identifier space. Peer identifiers (simply denoted ids in the following) are derived by applying some standard strong cryptographic hash function on peers intrinsic characteristics (see below). The value of $m$ (128 for the standard MD5 hash function) is chosen to be large enough to make the probability of identifiers collision negligible. The system is subject to churn, which is classically defined as the rate of turnover of peers in the system [6]. For scalability reasons, each peer knows only a small set of peers existing within the system and this knowledge generally varies according to the activity of the system. This set is typically called the node's local view. The particular algorithm used by peers to build their local view and to route messages induces the resulting overlay topology. Structured overlays (also called Distributed Hash Tables (DHTs)) build their topology according to structured graphs (e.g., hypercube, torus) as proposed in [9], [10], [11], [16], [17]. Peers self-organize within the structured graph according to a distance function $D$ based on peers ids, plus possibly other criteria such as geographical distance. Each data is assigned a unique identifier, called key, selected from the same $m$-bit identifiers space. Each peer $p$ owns a fraction of all the data items of the overlay. The mapping derives from the distance function $D$. In cluster-based overlays, clusters of peers substitute for peers at the vertices of the structured graph. Each vertex of the structured graph is composed of a set or cluster of peers. Peers join the clusters according to distance $D$. For instance in eQuus [18], peer $p$ joins the (unique) cluster whose members are geographically the closest to $p$, while in PeerCube [4], $p$ joins the (unique) cluster whose label is a prefix of $p$ 's identifier. Clusters in the overlay are uniquely labelled. Size of each cluster is both lower and upper bounded. The lower bound, named $C$ in the following, usually satisfies some constraint based on the assumed failure model. The upper bound, that we will call $S_{\max }$, is typically in $\mathcal{O}(\log N)$ where $N$ is the current number of peers in the overlay, to meet scalability requirements. Once a cluster size exceeds $S_{\max }$, this cluster splits into two smaller clusters, each one populated with the peers that are closer to each other according to distance $D$. Once a cluster undershoots its minimal size $C$, this cluster merges with the closest cluster in its neighborhood.

In the present work we assume that at cluster level, peers are organized as core and spare members. Members of the core set are primarily responsible for handling messages routing and clusters operations. Management of the core set is such that its size is maintained to constant $C$. Spare members are the complement number of peers in the cluster. Size $s$ of the spare set is such that $s \leq \Delta$ where $\Delta=S_{\max }-C$. In contrast to core members, spare members are not involved in any of the overlay operations. Rationale of this classification is two-fold: first it limits the management overhead caused by the natural churn present in typical overlay networks through the spare set management. Second it allows to introduce the unpredictability required to deal with malicious attacks through a randomized core set generation algorithm as shown in the sequel.

In the following we assume that join and leave events have an equal chance to occur in any cluster.

\section{B. Model of the Adversary}

A fundamental issue faced by any practical open system is the inevitable presence of peers that try to manipulate the system by exhibiting undesirable behaviors [13]. Such peers are classically called malicious or Byzantine. Malicious peers can devise complex strategies to prevent peers from discovering the correct mapping between peers and data keys. They can mount Sybil attacks [19] (i.e., an attacker generates numerous fake peers to pollute the system), they can do routing-table poisoning (also called eclipse attacks [8], [13]) by having honest peers redirecting outgoing links towards malicious ones, or they can simply drop or re-route messages towards other malicious peers. They can magnify their impact by colluding and coordinating their behavior. We model these strategies through a strong adversary that controls these malicious peers. A strong adversary is an adversary allowed to deviate arbitrarily far from the protocol specification. We assume that the adversary has 
large but bounded resources in that it cannot control more than a fraction $\mu(0<\mu<1)$ of malicious peers in the whole network. Note that in the following we make a difference between the whole universe and the overlay. The universe $\mathcal{U}$ encompasses all the peers that at some point may participate to the overlay (i.e. $2^{m}$ peers), while the overlay contains at any time the subset of participating peers (whose size is equal to $N)$. Thus, while $\mu$ represents the assumed fraction of malicious peers in the network, the goal of the adversary is to populate some parts of the overlay with a larger fraction of malicious peers in order to subvert the correct functioning of the overlay. Finally, a peer which always follows the prescribed protocols is said to be honest. Note that honest peers cannot a priori distinguish honest peers from malicious ones.

\section{Security Schemes}

We assume the existence of a public key cryptography scheme that allows each peer to verify the signature of each other peer. We also assume that correct peers never reveal their private keys. Peers ids and keys (private and public) are acquired via a registration authority. When describing the protocols, we ignore the fact that messages are signed, and recipients of a message ignore any message that is not signed properly. We also use cryptographic techniques to prevent a malicious peer from observing or unnoticeably modifying a message sent by a correct peer. However a malicious peer has complete control over the messages it sends and receives.

\section{Limited Sojourn Time and Random Distribution of ids}

As said in the Introduction, the two fundamental properties that prevent peers isolation are the guarantee that the distribution of peers identifiers is random, and that peers cannot stay forever at the same position in the system [5]. Both properties have been analytically proven assuming that the region size is kept constant [7].

To implement both limited sojourn time of the nodes at the same place in the overlay and unpredictable identifier assignment in a cluster-based overlay, we propose to limit the lifetime of peers identifiers and to randomize their computation. Specifically, peers identifiers are initially generated based on certificates acquired at trustworthy Certification Authorities (CAs). Initial identifiers (denoted $i d^{0}$ ) are generated as the result of applying a hash function $\mathcal{H}$ to some of the fields of a X.509 [20] certificate. To enforce all peers, including malicious ones, to leave and rejoin the system from time to time, we add the creation date $t_{0}$ to the fields that appear in the peer certificate that will be hashed to generate the peer identifier (note that by the properties of hash functions, this guarantees that peers identifiers are unpredictable). We limit the lifetime peers identifier through an incarnation number. The current incarnation $k$ of any peer is given by the following expression $k=\left\lceil\left(t-t_{0}\right) / L\right\rceil$, where $t_{0}$ is the initial creation time of the peer's certificate, $t$ is the current time, and $L$ is the length of the lifetime of peers incarnation. Thus, the $k^{t h}$ incarnation of a peer $p$ expires when $p$ local clock reads $t_{0}+k L$. At this time $p$ must rejoin the system using its $(k+1)^{t h}$ incarnation. The $t_{0}$ parameter is one of the fields in the peer's certificate and since certificates are signed by the CA, it cannot be unnoticeably modified by a malicious peer. Moreover, a certificate commonly contains the public key of the certified entity. This way, messages exchanged by the peers can be signed using the sender private key, preventing malicious peers from unnoticeably altering messages originated from other peers in the system. Messages must contain the certificate of their issuer, so as to allow recipients to validate them. ${ }^{1}$

Therefore, at any time, any peer can check the validity of the identifier of any other peer $q$ in the system, by simply calculating the current incarnation $k$ of peer $q$ and generating the corresponding identifier. Specifically, the current identifier of peer $q$, denoted in the following as $i d_{q}$, is calculated by hashing $q$ initial identifier $\left(i d_{q}^{0}\right)$ with the current incarnation $k$ of $q$, i.e., $i d_{q}=\mathcal{H}\left(i d_{q}^{0} \times k\right)$. This leads to the following property.

Property 1 (Limited Sojourn Time): Let $\mathcal{D}$ be some cluster of the system and $p$ some peer in the overlay. Then $q$ belongs to $\mathcal{D}$ at time $t$ if only if $i d_{q}$ matches the label of $\mathcal{D}$ according to distance $D$ (we say that $q$ is valid for $\mathcal{D}$ ).

If some peer $p$ detects that the id of one of its neighbors $q$ is not valid then it cuts its connection with $q$. Peer $q$ may re-join the overlay by triggering a join operation at cluster $\mathcal{D}^{\prime}$ such that $i d_{q}$ is valid for $\mathcal{D}^{\prime}$. Note that because clocks are loosely synchronized, it is possible that a correct peer is still using its id for incarnation $k$ when other correct peers would expect it to be in incarnation $k+1$. To mitigate this problem, we assume that any correct peer may have two subsequent valid incarnation numbers, for a fixed grace window $W$ of time that encompasses the expiration time of an incarnation number ( $W$ is the maximum deviation of the clocks of any two correct peers). More precisely, at any time $t$, both incarnation $k$ and $k^{\prime}$ are valid, where $k=\lceil(t-$ $\left.\left.W / 2-t_{0}\right) / L\right\rceil$, and $k^{\prime}=\left\lceil\left(t+W / 2-t_{0}\right) / L\right\rceil$. Thus, although each peer $p$ has, at any time $t$, a single incarnation number that it uses to define its current id, other peers calculate two possible incarnation numbers for $p$. These are frequently equal, but may differ when $p$ 's local time is close to the expiration time of its current/last incarnation.

\section{OpERATIONS OF THE OVERLAY}

To protect the system against the presence of malicious peers in the overlay, we propose to take advantage of peers role separation at cluster level to design robust operations.

\footnotetext{
${ }^{1}$ Note that there are means to optimize this procedure as for example by exchanging certificates during an initialization phase. However this is out of the scope of our paper.
} 
Briefly, the join operation is designed so that brute force denial of service attacks are discouraged. The Leave operation impedes the adversary from predicting what is going to be the composition of the core set after a given sequence of join and leave events triggered by its malicious peers. Finally, as both merge and split operations induce topological changes in the overlay, and more importantly may have an influence on the subset of the identifier space the adversary may gain control over, ${ }^{2}$ these operations have been designed so that the adversary has, in expectation, no interest to trigger them. Specifically,

- join(p): when a peer $p$ joins the system, it joins the spare set of the closest cluster in the system (according to distance $D$ ). Core members of this cluster update their spare view to reflect $p$ 's insertion (note that the spare view update does not need to be tightly synchronized at all core members).

- leave(p): When a peer $p$ leaves a cluster either $p$ belongs to the spare set or to the core set. In the former case, core members simply update their spare view to reflect $p$ 's departure, while in the latter case, the core view maintenance procedure is triggered. This procedure consists in replacing $k-1$ randomly chosen members of the core set with $k$ peers randomly chosen from the whole cluster, where $1 \leq k \leq C$ (recall that $C$ is the size of the core set, cf. Section III-A).

- split $(\mathcal{D})$ : when a cluster $\mathcal{D}$ has reached the conditions to split into two smaller clusters $\mathcal{D}^{\prime}$ and $\mathcal{D}^{\prime \prime}$, core sets of both $\mathcal{D}^{\prime}$ and $\mathcal{D}^{\prime \prime}$ are built. Priority is given to core members of $\mathcal{D}$ and completion is done with randomly chosen spares in $\mathcal{D}$. This random choice is handled through a Byzantine-tolerant consensus run among core members of $\mathcal{D}$. Spares members of $\mathcal{D}^{\prime}$ (resp. $\mathcal{D}^{\prime \prime}$ ) are populated with the remaining spares members of $\mathcal{D}$ that are closer to $\mathcal{D}^{\prime}$ than to $\mathcal{D}^{\prime \prime}$ (resp. closer to $\mathcal{D}^{\prime \prime}$ than to $\mathcal{D}^{\prime}$ ).

- merge $\left(\mathcal{D}^{\prime}, \mathcal{D}^{\prime \prime}\right)$ : when some cluster $\mathcal{D}^{\prime}$ has reached the conditions to merge (i.e., its spare set is empty), it merges with the closest cluster $\mathcal{D}^{\prime \prime}$ to $\mathcal{D}^{\prime}$. The created cluster $\mathcal{D}$ is composed by a core set whose members are the core set members of $\mathcal{D}^{\prime \prime}$ and by a spare set whose members are the union of the spare members of $\mathcal{D}^{\prime \prime}$ and the core set members of $\mathcal{D}^{\prime}$.

These four operations make up the overlay protocol. In the following protocol $_{k}$ will refer to as these four operations with $1 \leq k \leq C$ the amount of randomization of the core view maintenance procedure of the leave operation.

\section{Specification of the Adversarial Behavior}

Based on the operations described in the previous section, we investigate how malicious peers could proceed

\footnotetext{
${ }^{2}$ Indeed, a merge operation doubles the subset of the identifier space a cluster is responsible for, while a split operation divides it per two.
}

to compromise correctness of a targeted cluster. Clusters correctness is jeopardized as soon as a quorum of its core members are malicious. It is well known that a necessary and sufficient condition to prevent agreement among a set of nodes is that strictly more than a third of the population set is malicious [21]. In our context, cluster $\mathcal{D}$ is said to be polluted if its core set is populated by more than a quorum $c$ of malicious peers where $c=\lfloor(C-1) / 3\rfloor$ malicious peers. Otherwise, this cluster $\mathcal{D}$ is said to be safe.

\section{A. Increasing Global Representation of Malicious Identifiers}

As a consequence of assigning an initial unique random id from an $m$-bit identifier space to peers and of periodically pushing peers to random regions in the overlay, the strategy of the adversary to increase the global representation of malicious identifiers is a combination of the following three actions.

First, the adversary maximizes the number of malicious peers that sit in the whole overlay. By doing so, the adversary augments the likelihood for its malicious peers to join targeted clusters. For those peers which cannot immediately enter targeted clusters (because their current ids do not match the targeted clusters label), but rather join different clusters, the goal of the adversary is to pollute these clusters as well. This augments the subset of identifiers space the adversary has gained control over, and thus empowers it to progressively surround the targeted clusters.

Second, the adversary may in some specific cases decide to trigger a leave operation for some malicious peer in a given core set if the outcome of the operation increases the global representation of malicious identifiers in that set. Indeed, if by having a malicious peer leaving the core set of a cluster, the likelihood that the outcome of the core set maintenance process strictly increases the number of malicious peers in the renewed core set, then the adversary triggers a leave operation. Formally,

Rule 1 (Adversarial Leave Strategy): Let $\mathcal{D}$ be a cluster such that at time $t$ its core set $\mathcal{C}$ contains $0<x \leq c$ valid malicious peers and its spare set contains $y>1$ valid malicious peers. At time $t$ the adversary triggers a leave $(p)$ operation for malicious peer $p$ in $\mathcal{C}$ if, for a given small positive threshold $\nu$

$$
\sum_{j=x+1}^{x-1+\min (k, y)} \mathbb{P}\left\{\begin{array}{c}
\text { exactly } j \text { malicious peers } \in \mathcal{C} \\
\text { after the leave operation }
\end{array}\right\}>1-\nu .
$$

Recall that $k$ is the amount of randomization of the leave operation. Note that for $k=1$, Relation (1) is never satisfied. Thus in this specific situation, there is no incentive for malicious peers to trigger voluntary leaves. For $k>1$, malicious peers collude together to force the one among them (whose id will expire the soonest) to leave the core. As the experiments will show, decreasing the amount of randomization of the leave operation provides the best strategy against targeted attacks. 
Finally, once the adversary has succeeded in polluting a cluster $\mathcal{D}$, he must minimize the likelihood that $\mathcal{D}$ switches back to a safe state. Switching to a safe state may occur subsequent to either the core maintenance procedure, the merge, or the split operations. The two latter cases are detailed in the following section. Regarding the former case, the adversary can bias the core set maintenance procedure by replacing the left peer with a (valid) malicious peer from the spare set if any. On the other hand, if the spare set does not contain any malicious peers then the adversary has no choice other than choosing a honest peer (otherwise clusters in $\mathcal{D}$ vicinity will quickly detect that the size of $\mathcal{D}$ core set is less than $C$ ).

\section{B. Decreasing the Occurrence of Topological Operations}

So far we have seen that the adversary triggers leave operations for its malicious peers if with high probability it increases the population of malicious peers in the core set. However, the adversary will trigger such departures only if this does not lead the cluster to merge with another cluster. Indeed, by construction of the merge operation (cf. Section IV), when $\mathcal{D}$ core members trigger a merge with their closest neighbor $\mathcal{D}^{\prime}$ then all $\mathcal{D}$ members are pushed to the spare set of the new created cluster $\mathcal{D}^{\prime \prime}$ while core members of $\mathcal{D}^{\prime}$ keep their status of core members in $\mathcal{D}^{\prime \prime}$. This clearly deters the adversary from triggering merge operations. We have also seen that to gain the control of clusters, the strategy of the adversary is to maximize the number of malicious peers in both the core and the spare sets of any cluster. However once a cluster is polluted, the adversary has no interest to let this cluster grow in such a way that this cluster will undergo a split operation. Indeed, the outcome of a split operation cannot increase the subset of identifiers space the adversary has gained control over-at best, it keeps it the same. Thus when a polluted cluster is close to split, the adversary acts so that no join operations are triggered. Note that the adversary can prevent honest peers from joining $\mathcal{D}$ whenever $s>1$. This guarantees that $\mathcal{D}$ will not grow because of honest peers, while ensuring that $\mathcal{D}$ will not undergo a merge operation as much as possible. Specifically,

Rule 2 (Adversarial Join Strategy): Let $\mathcal{D}$ be a cluster such that at time $t$ its core set contains $\ell>c$ valid malicious peers. Any join event issued by peer $q$ and received at $\mathcal{D}$ at time $t$ is discarded if ( $q$ is honest and $s>1$ ) or $(s=\Delta-1)$. Recall that $\Delta$ represents the maximal size of the spare set (c.f. Section III-A).

A possible implementation of Rule 2 is as follows: upon receipt of a join event from an honest peer $q$, the adversary asks the malicious core members to positively acknowledge $q$, so that $q$ does not detect that $\mathcal{D}$ is polluted, however the associated join operation is not triggered.

To summarize, the strategy of the adversary is to maximize the whole subset of the identifiers space it has gained control over. This is achieved by first never asking its malicious peers to leave their cluster except if either Property 1 does not hold or Rule 1 holds, and second by having the maximal number of malicious peers join the system except if Rule 2 holds.

\section{Modeling the AdVERs arial Strategy}

The evolution of any given cluster $\mathcal{D}$ follows both the overlay protocol protocol $_{k}$ (cf. Section IV) and the strategy of the adversary (cf. Section V). To analyze the impact of the adversarial strategy, we make a difference between join (resp. leave) events and join (resp. leave) operations. Indeed, from above, the number of leave and join events issued at malicious peers is greater than or equal to the number of the associated leave and join operations.

We model the effect of join and leave events using a homogeneous discrete-time Markov chain denoted by $X=\left\{X_{n}, n \geq 0\right\}$. Markov chain $X$ represents the evolution of the number of malicious peers in both the core set and the spare set of cluster $\mathcal{D}$. The state space $\Omega$ of $X$ is defined by $\Omega=\{(s, x, y) \mid 0 \leq s \leq \Delta, 0 \leq x \leq C, 0 \leq y \leq s\}$. For $n \geq 1$, the event $X_{n}=(s, x, y)$ means that, after the $n$-th transition (i.e., the $n$-th join or leave event), the size of the spare set is equal to $s$, the number of malicious peers in the core set is equal to $x$ and the number of malicious peers in the spare set is equal to $y$. In the remaining of the paper, the initial probability distribution of $X$ is denoted by $\alpha$. The transition probability matrix $M$ of $X$ is detailed below.

We define a state as polluted if in this state the core set contains more than $c=\lfloor(C-1) / 3\rfloor$ malicious peers. Conversely, a state that is not polluted is said to be safe.

The subset of safe states, denoted by $S$, is defined by $S=\{(s, x, y) \mid 0<s<\Delta, 0 \leq x \leq c, 0 \leq y \leq s\}$, while the subset of polluted states, denoted by $P$, is defined by $P=\{(s, x, y) \mid 0<s<\Delta, c+1 \leq x \leq C, 0 \leq$ $y \leq s\}$. The system alternates between safe and polluted states until entering closed states. Closed states represent the logical disappearance of cluster $\mathcal{D}$ from the graph. This occurs when either $\mathcal{D}$ splits into two smaller clusters (i.e., its spare set has reached its maximal size $\Delta$ ) or $\mathcal{D}$ merges with its closest neighbor (i.e., the size of its spare set has shrunk to 0 ). Three sets of closed states exist. The set of safe merge closed states $A_{S}^{m}$ defined as $A_{S}^{m}=\{(s, x, y) \mid$ $(s=0) \wedge(0 \leq x \leq c)\}$, the set of safe split closed states $A_{S}^{\ell}$ defined as $A_{S}^{\ell}=\{(s, x, y) \mid(s=\Delta) \wedge(0 \leq x \leq c)\}$, and the set of polluted merge closed states $A_{P}^{m}$ defined as $A_{P}^{m}=\{(s, x, y) \mid(s=0) \wedge(c+1 \leq x \leq C)\}$. Note that by Rule 2 the states such that $s=\Delta$ and $c+1 \leq x \leq C$ are not reachable because the adversary strategizes to prevent a polluted cluster from triggering a split operation (cf. Section V-B). As a consequence the set of polluted split closed states is empty. Matrix $P$ is partitioned with respect 
to the decomposition of $\Omega=S \cup P \cup A_{S}^{m} \cup A_{S}^{\ell} \cup A_{P}^{m}$

$$
M=\left(\begin{array}{ccccc}
M_{S} & M_{S P} & M_{S A_{S}^{m}} & M_{S A_{S}^{\ell}} & M_{S A_{P}^{m}} \\
M_{P S} & M_{P} & M_{P A_{S}^{m}} & M_{P A_{S}^{\ell}} & M_{P A_{P}^{m}} \\
0 & 0 & M_{A_{S}^{m}} & 0 & 0 \\
0 & 0 & 0 & M_{A_{S}^{\ell}} & 0 \\
0 & 0 & 0 & 0 & M_{A_{P}^{m}}
\end{array}\right)
$$

where $M_{U V}$ is the sub-matrix of dimension $|U| \times|V|$ containing the transitions from states of $U$ to states of $V$, with $U, V \in\left\{S, P, A_{S}^{m}, A_{S}^{\ell}, A_{P}^{m}\right\}$. We simply write $M_{U}$ for $M_{U U}$. Note that $A_{S}^{m}, A_{S}^{\ell}$, and $A_{P}^{m}$ are absorbing classes. In the same way, the initial probability distribution $\alpha$ is partitioned by writing $\alpha=\left(\begin{array}{lllll}\alpha_{S} & \alpha_{P} & \alpha_{A_{S}^{m}} & \alpha_{A_{S}^{\ell}} & \alpha_{A_{P}^{m}}\end{array}\right)$, where sub-vector $\alpha_{U}$ contains the initial probabilities of states $U \in\left\{S, P, A_{S}^{m}, A_{S}^{\ell}, A_{P}^{m}\right\}$. Figure 1 depicts an aggregated view of the states partition of process $X$.

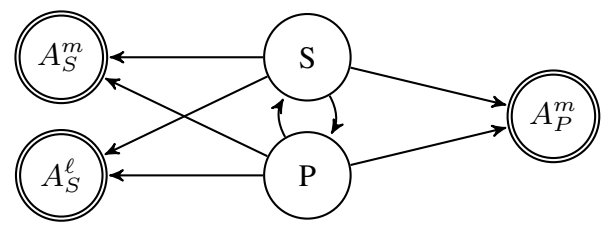

Figure 1: Aggregated view of Markov chain $X$. All the transient safe and polluted states are respectively represented by $S$ and $P$. States $A_{S}^{m}, A_{S}^{\ell}$ and $A_{P}^{m}$ represent all the closed safe and polluted states. For $C=7$ and $\Delta=7$, we have 288 states.

Computation of transition matrix $M$ is illustrated in Figure 2. In this tree, each edge is labelled by a probability and each leaf represents the state of the cluster. This figure shows all the states that can be reached from state $(s, x, y)$ and the corresponding transition probabilities. Transition probabilities depend on $i$ ) the type of operation that occurred (join or leave operation from the core or the spare set), ii) the type of peers involved in this operation (honest or malicious), and iii) the ratio of malicious peers already present in the core set. The probability associated with each one of these states is obtained by summing the products of the probabilities discovered along each path starting from the root to the leaf corresponding to the target state. For instance, the leftmost branch of the tree corresponds to the scenario in which some malicious peer wishes to join the polluted cluster $\mathcal{D}$. By Rule 2, this peer successfully joins the cluster, leading to state $(s+1, x, y+1)$. Now if we consider the rightmost branch in the tree, this represents the situation in which cluster $\mathcal{D}$ is polluted and one of its malicious core member $p$ is no more valid. By Property 1, $p$ leaves $\mathcal{D}$, however as $\mathcal{D}$ is polluted, the adversary bias the core management procedure by replacing $p$ with another malicious peer from $\mathcal{D}$ spare set. State $(s-1, x, y-1)$ is reached. For space reasons, derivation of the transition probability matrix $M$ is presented in [22].

Modeling and computation of both Property 1 and Rule 1 are as follows. Regarding Property 1 , let $d$ be the probability (per unit of time) that the limited lifetime of some peer $p$ has not expired. Hence $d$ is homogeneous to a frequency, and $d \times \Delta t$ represents the probability that the lifetime of a given peer identifier has not expired during $\Delta t$ units of time. From a practical point of view, we model the limited lifetime of a peer identifier as an exponential decay process whose halftime constant (denoted $t_{1 / 2}$ ) is related to $d$ by the standard relation $t_{1 / 2}=\ln 2 /(1-d)$. Consequently, the value of $L$ (cf. III-D) might be calibrated so that $99 \%$ of a given population has decayed after $L$ unit of time. Setting $L=$ $6.65 \times t_{1 / 2}$ satisfies this requirement ${ }^{3}$. Then the probability that for all the peers belonging to a set of size $z$ Property 1 holds is equal to $d^{z}$. Regarding Rule 1 , let $q(k, \ell, u, v)$ be the probability of getting $u$ red balls when $k$ balls are drawn without replacement from an urn containing $v$ red balls and $\ell-v$ white balls. We have $q(k, \ell, u, v)=\left(\begin{array}{l}v \\ u\end{array}\right)\left(\begin{array}{l}\ell-v \\ k-u\end{array}\right) /\left(\begin{array}{l}\ell \\ k\end{array}\right)$. $q($.$) is referred to as the hypergeometric distribution. Let$ $(s, x, y)$ be the current state of the Markov chain associated to cluster $\mathcal{D}$. Then Rule 1 holds if the chain enters one of the following states $(s-1, x+1, y-2), \ldots,\left(s-1, x^{\prime}, y^{\prime}\right)$, with $x^{\prime}=x+y-1$ and $y^{\prime}=0$ if $k \geq x+y-1$ otherwise $x^{\prime}=k$ and $y^{\prime}=x+y-1-k$, right after the voluntary departure of one malicious valid core member with probability $1-\nu$. From the leave operation Relation (1) writes as

$\sum_{i=i_{0}}^{i_{\max }} \sum_{j=i+2}^{j_{\max }} q(k-1, C-1, i, x-1) q(k, s+k-1, j, y+i)>1-\nu$.

where $i_{0}=\max (0, k-1-(C-x)), i_{\max }=\min (k-1, x-1)$ and $j_{\max }=\min (k, y+i)$. In Figure 2, notation " $1_{\{(2)\}}$ " means that Relation (2) holds.

\section{STUdy OF A Cluster BeHAVIOR IN AN ADVERSARIAL SETTING}

In this section, we study the behavior of a cluster according to the power of the adversary, the frequency of the induced churn, and the amount of randomization $k$ introduced in protocol $_{k}$.

\section{A. Initial Distributions}

In the experiments conducted for this work, we consider two initial distributions. The first one, which we denote by $\beta$, consists in assuming that the initial size of the spare set (denoted as $s_{0}$ ) is uniformly distributed on $\{1, \ldots, \Delta-1\}$. The initial number $C_{0}$ of malicious peers in the core set and the one $S_{0}$ in the spare set both follow a binomial distribution. The initial state $X_{0}$ is thus defined by $X_{0}=$ $\left(s_{0}, C_{0}, S_{0}\right)$. Assuming that $C_{0}$ and $S_{0}$ are independent, we get for $x=0, \ldots, C$ and $y=0, \ldots, s_{0}$

$$
\begin{aligned}
\beta(x, y) & =\mathbb{P}\left\{C_{0}=x, S_{0}=y\right\} \\
& =\left(\begin{array}{c}
C \\
x
\end{array}\right) \mu^{x}(1-\mu)^{C-x}\left(\begin{array}{c}
s_{0} \\
y
\end{array}\right) \mu^{y}(1-\mu)^{s_{0}-y}(3)
\end{aligned}
$$

\footnotetext{
${ }^{3}$ We have that $6.65 \geq \ln 100 / \ln 2$.
} 
(s, X,y)

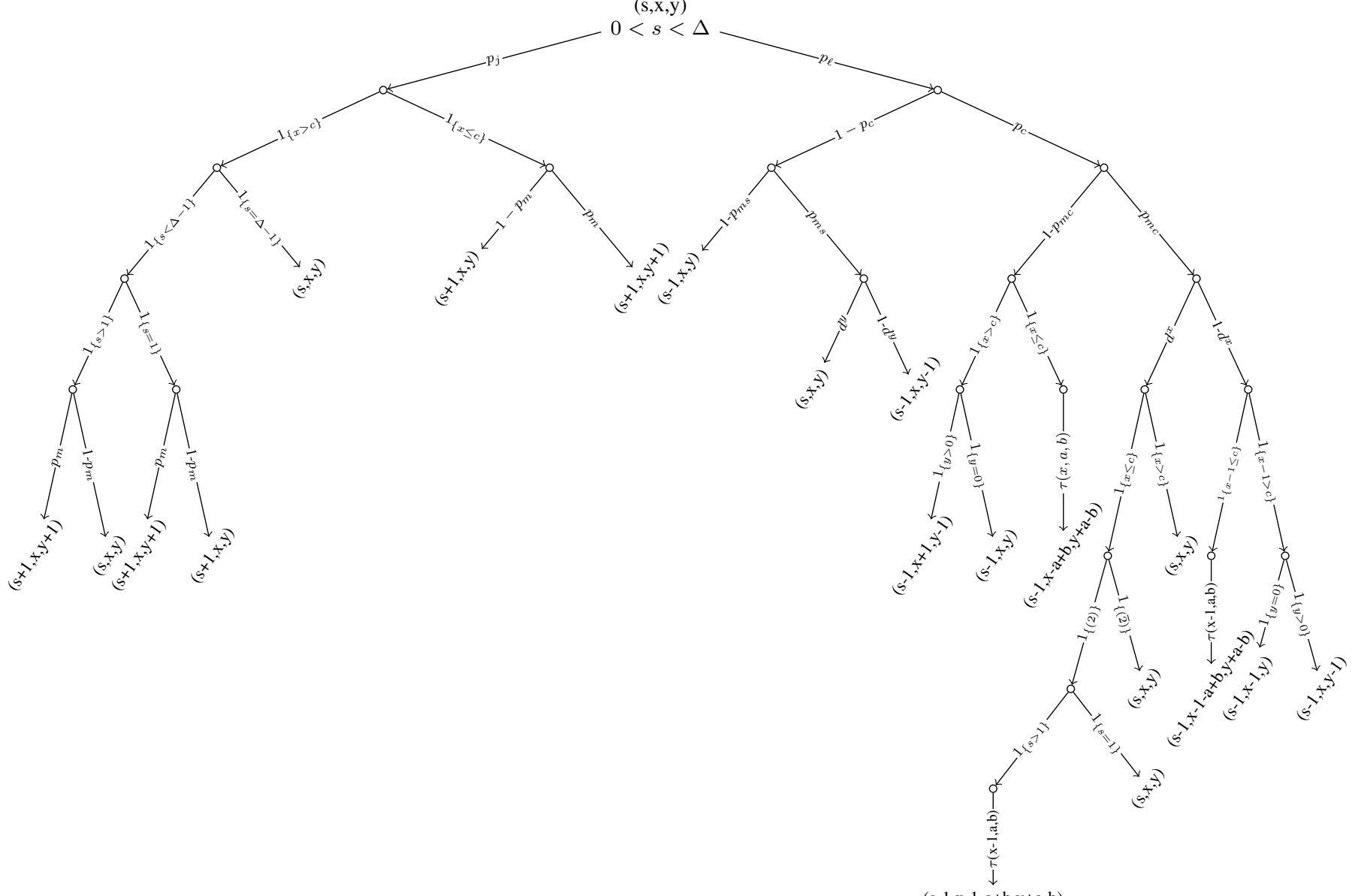

$(\mathrm{s}-1, \mathrm{x}-1-\mathrm{a}+\mathrm{b}, \mathrm{y}+\mathrm{a}-\mathrm{b})$

\begin{tabular}{|c|c|c|}
\hline Probabilities & Value & Meaning of the probability \\
\hline$\mu$ & & ratio of Byzantine peers in the universe $\mathcal{U}$ \\
\hline$S_{\max }$ & & maximal size of a cluster \\
\hline$C$ & & size of the core set of a cluster ( $C$ is a system parameter) \\
\hline$\Delta$ & $S_{\max }-C$ & maximal size of the spare set of a cluster \\
\hline$s$ & & current size of the spare set \\
\hline$x$ & & number of malicious peers in the core set \\
\hline$y$ & & number of malicious peers in the core set \\
\hline$d$ & & $\begin{array}{l}\text { probability that the lifetime of a given peer identifier has not expired } \\
\text { (per unit of time) }\end{array}$ \\
\hline$t_{1 / 2}$ & $\ln 2 /(1-d)$ & half-life of a peer identifier \\
\hline$p_{j}\left(\right.$ resp. $\left.p_{\ell}\right)$ & $1 / 2$ & join (resp. leave) event probability \\
\hline$p_{c}$ & $C /(C+s)$ & probability for a peer to belong to the cluster core set \\
\hline$p_{m}$ & $\mu$ & probability that the joined peer is malicious \\
\hline$p_{m c}$ & $x / C$ & probability for a core member to be malicious \\
\hline$p_{m s}$ & $y / s$ & probability for a spare member to be malicious \\
\hline $1-d^{x}$ & & probability that Property 1 is satisfied in the core set during one unit of time \\
\hline $1-d^{y}$ & & probability that Property 1 is satisfied in the spare set during one unit of time \\
\hline $1_{\{A\}}$ & 1 if condition $A$ is true, 0 otherwise & represents the indicator function \\
\hline$\tau(x, a, b)$ & $\begin{array}{l}q(k-1, C-1, a, x) q(k, s+k-1, b, y+a) \\
\text { with } q(k, \ell, u, v)=\left(\begin{array}{l}v \\
u\end{array}\right)\left(\begin{array}{l}\ell-v \\
k-u\end{array}\right) /\left(\begin{array}{l}\ell \\
k\end{array}\right)\end{array}$ & $\begin{array}{l}\text { probability of building the core (resp. spare) set with } x-a+b \\
\text { (resp. } y-b+a) \text { malicious peers where } \\
\max (0, k-1-(C-1-x)) \leq a \leq \min (x, k-1) \text {, and } \\
\max (0, k-(s+k-1-(y+a)) \leq b \leq \min (y+a, k)\end{array}$ \\
\hline
\end{tabular}

Figure 2: Transition diagram for the computation of the transition probability matrix $M$. 
The second one, that we denote by $\delta$, consists in starting from state $\left(s_{0}, 0,0\right)$, that is the state free from malicious peers, where $s_{0}=\lfloor\Delta / 2\rfloor$. We have

$$
\delta\left(s_{0}, x, y\right)=1_{\{x=0, y=0\}} .
$$

\section{B. Time Spent by Protocol $_{k}$ in Safe States}

As described in Section VI the Markov chain $X$ is reducible, the subset of states $S$ and $P$ are transient and the subsets $A_{S}^{m}, A_{S}^{\ell}$ and $A_{P}^{m}$ are closed subsets. We start our study by first investigating the distribution of $T_{S}^{(k)}$ which counts the total time spent by protocol $_{k}$ in the subset of safe states $S$ before absorption. Specifically $T_{S}^{(k)}=\sum_{n=0}^{\infty} 1_{\left\{X_{n} \in S\right\}}$. Following the results in [23], the expectation of $T_{S}^{(k)}$ is given by

$$
E\left(T_{S}^{(k)}\right)=v(I-R)^{-1} \mathbb{1} .
$$

where $v=\alpha_{S}+\alpha_{P}\left(I-M_{P}\right)^{-1} M_{P S}$ and $R=M_{S}+$ $M_{S P}\left(I-M_{P}\right)^{-1} M_{P S}$.

\section{Time Spent by Protocol $_{k}$ in Polluted States}

In the same way, its expectation is given by

$$
E\left(T_{P}^{(k)}\right)=w(I-Q)^{-1} \mathbb{1}
$$

where $w=\alpha_{P}+\alpha_{S}\left(I-M_{S}\right)^{-1} M_{S P}$ and $Q=M_{P}+$ $M_{P S}\left(I-M_{S}\right)^{-1} M_{S P}$.

Figure 3 compares the expected number of operations spent in safe and polluted states before absorption for two protocols, protocol $_{1}$ and protocol $_{C}$, as a function of $\mu, d$ and the two initial distributions $\delta$ and $\beta$. We have only detailed protocols 1 and $C$ (here $C=7$ ) as we have observed that they bound the performance of the other ones (here, the

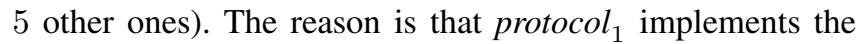
least amount of randomization of the leave operation while protocol $_{C}$ implements the largest one. This will allow us to illustrate the fact that, counterintuitively, increasing the amount of randomization of the operations does not make them necessarily more resilient to strong adversaries.

Lessons Learnt from these Experiments: The first lesson that we can draw from this figure is the impact of the initial distribution on the behavior of the cluster. When this distribution equals $\delta$ (i.e., the cluster is initially free from malicious peers), the number of operations run in safe states is much larger than the one spent in polluted ones for both protocols. This holds even for very large values of both $\mu$ and $d$. This comes from the combined effects of both the join and leave operations. The former one prevents new peers to directly belong to the core set, while the latter one randomizes the core set population, demanding accordingly a certain amount of time for the adversary to successfully pollute a cluster. On the other hand, when starting with $\alpha=\beta$, both the core and the spare sets are initially populated with malicious peers (proportionally to $\mu$ ). Hence this requires less effort for the adversary to gain control of the cluster.

The second lesson relates to the impact of randomization on cluster resiliency. For a given initial distribution $\alpha$, protocol $_{1}$ always outperforms protocol $_{C}$, that is for both a given $\mu$ and a given $d, E\left(T_{S}^{(1)}\right) \geq E\left(T_{S}^{(C)}\right)$ and $E\left(T_{P}^{(1)}\right) \leq E\left(T_{P}^{(C)}\right)$. This shows that increasing the amount of randomization does not make the protocols more resilient to targeted attacks.

The third lesson is linked to peers identifiers lifetime $d$. For increasing values of $d$, the expected time spent in safe clusters strictly increases, while the one spent in polluted clusters slightly decreases, for a fixed $\mu$. This is explained by the fact that malicious peers can stay longer in the cluster, increasing accordingly their proportion in the cluster up to pollution. Once polluted, the adversary prevents split operations from occurring. This increases accordingly the time spent in polluted states and thus the lifetime of the cluster. Clearly this phenomenon is more noticeable for larger values of $\mu$. Now for $d$ close to 1 , malicious peers are allowed to stay almost forever in their cluster. This enables them to quickly gain the quorum in their cluster, and thus prevent any safe operation to be triggered even for very small values of $\mu$ (see Table I). This clearly confirms that churn is a fundamental ingredient to defend against targeted attacks.

Finally, it is interesting to remark that in a failure free environment $(\mu=0)$, we have $E\left(T_{S}^{(k)}\right)+E\left(T_{P}^{(k)}\right)=$ $\left\lfloor\Delta^{2} / 4\right\rfloor=12$. Actually $\left\lfloor\Delta^{2} / 4\right\rfloor$ corresponds to the maximal expected number of steps before absorption in a one dimensional random walk with borders (here, $\Delta$ is the length between the two borders).

D. Successive Times Spent by Protocol $_{k}$ in Polluted and Safe States

A deeper investigation of protocol $_{k}$ can be conducted by studying the duration and frequency of successive times spent in subsets $S$ and $P$. For $n \geq 1$, we denote by $T_{S, n}^{(k)}$ (resp. $T_{P, n}^{(k)}$ ) the distribution of the time spent by the Markov chain $X$ during its $n$-th sojourn in subset $S$ (resp. $P$ ). Thus the total time spent in subset $S$ (resp. $P$ ) before reaching subsets $A_{S}$ and $A_{P}$ is given by

$$
T_{S}^{(k)}=\sum_{n=1}^{\infty} T_{S, n}^{(k)} \text { and } T_{P}^{(k)}=\sum_{n=1}^{\infty} T_{P, n}^{(k)} .
$$

From [24], we have for $n \geq 1$ and $\ell \geq 0$

$$
E\left(T_{S, n}^{(k)}\right)=v G^{n-1}\left(I-M_{S}\right)^{-1} \mathbb{1},
$$

where $v$ is defined in Relation (5) and $G=(I-$ $\left.M_{S}\right)^{-1} M_{S P}\left(I-M_{P}\right)^{-1} M_{P S}$, and

$$
E\left(T_{P, n}^{(k)}\right)=w H^{n-1}\left(I-M_{P}\right)^{-1} \mathbb{1},
$$

where $w$ is defined in Relation 6 and $H=(I-$ $\left.M_{P}\right)^{-1} M_{P S}\left(I-M_{S}\right)^{-1} M_{S P}$. 

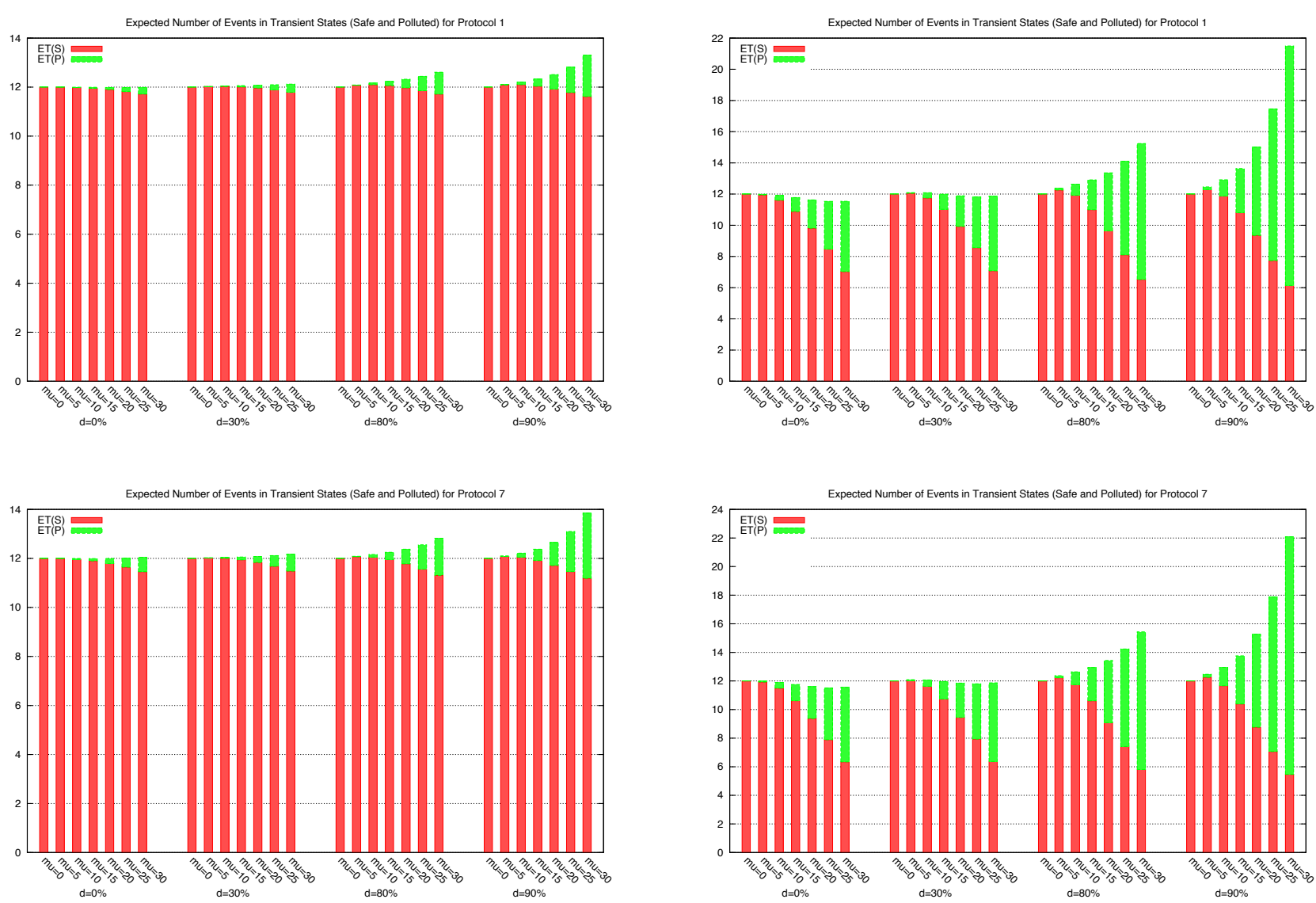

Figure 3: $E\left(T_{S}^{(k)}\right)$ (Relation (5)) represented by hatched bars, and $E\left(T_{P}^{(k)}\right)$ (Relation (6)) represented by plain bars as a function of $k, \mu$ and $d$. In all these experiments $C=7, \Delta=7$, and for the two figures on the left (resp. right), we have $\alpha=\delta($ resp. $\alpha=\beta)$.

\begin{tabular}{|c|c|c|c|c|c|c|c|c|c|c|c|c|}
\hline & \multicolumn{3}{|c|}{$\mu=0 \%$} & \multicolumn{3}{c|}{$\mu=10 \%$} & \multicolumn{3}{c|}{$\mu=20 \%$} & \multicolumn{3}{c|}{$\mu=30 \%$} \\
\hline$d$ & 0.95 & 0.99 & 0.999 & 0.95 & 0.99 & 0.999 & 0.95 & 0.99 & 0.999 & 0.95 & 0.99 & 0.999 \\
\hline$E\left(T_{S}^{(1)}\right)$ & 12.0 & 12.0 & 12.0 & 12.09 & 12.08 & 12.08 & 11.88 & 11.84 & 11.83 & 11.54 & 11.48 & 11.47 \\
$E\left(T_{P}^{(1)}\right)$ & 0.0 & 0.0 & 0.0 & 0.15 & 2.6 & 1518 & 1.14 & 699.7 & 511810822. & 5.96 & 12597. & 9299884149 \\
\hline
\end{tabular}

Table I: $E\left(T_{S}^{(k)}\right)$ and $E\left(T_{S}^{(k)}\right)$ as a function of $\mu$ and $d$. In these experiments $k=1, C=7, \Delta=7$, and $\alpha=\delta$.

\begin{tabular}{|c|c|c|c|c|}
\hline & $\mu=0 \%$ & $\mu=10 \%$ & $\mu=20 \%$ & $\mu=30 \%$ \\
\hline$E\left(T_{S, 1}^{(1)}\right)$ & 12 & 12.085 & 11.890 & 11.570 \\
$E\left(T_{S, 2}^{(1)}\right)$ & 0 & 0.013 & 0.033 & 0.043 \\
$E\left(T_{P, 1}^{(1)}\right)$ & 0 & 0.099 & 0.558 & 1.611 \\
$E\left(T_{P, 2}^{(1)}\right)$ & 0 & 0.004 & 0.26 & 0.075 \\
\hline
\end{tabular}

is the protocol does not alternate between safe and polluted states. This is very noticeable for small values of $\mu$ while a little bit less for larger ones.

Table II: Successive sojourn times in transient states $S$ and $P$ as a function of $\mu$ and $d$. In these experiments $k=1$, $C=7, \Delta=7, d=90 \%$, and $\alpha=\delta$.

Table II shows the expected duration of successive sojourn times in subsets $S$ and $P$ for protocol $_{k}$, with $k=1$. We can see that $E\left(T_{S}^{(k)}\right) \simeq E\left(T_{S, 1}^{(k)}\right)$ and $E\left(T_{P}^{(k)}\right) \simeq E\left(T_{P, 1}^{(k)}\right)$, that

\section{E. Absorption Probabilities}

Clusters eventually either split into two smaller clusters or merge with another cluster. An important question to be answered is whether or not split operations are more frequent than merge ones (for safe clusters), and whether or not polluted clusters merge more frequently than safe 

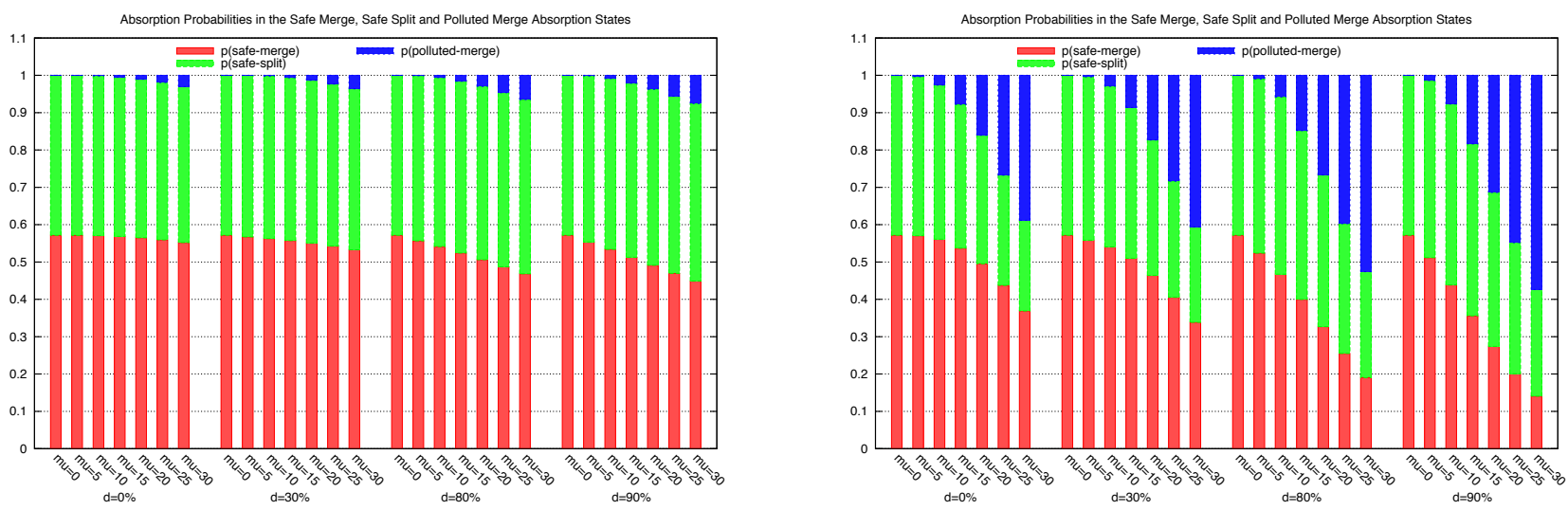

Figure 4: $p\left(A_{S}^{m}\right), p\left(A_{S}^{\ell}\right)$ and $p\left(A_{P}^{m}\right)$ (cf. Relation (9)) respectively represented by red hatched, plain and blue hatched bars as a function of $\mu$ and $d$. In these experiments $k=1, C=7$, and $\Delta=7$. We have $\alpha=\delta$ for the left figure and $\alpha=\beta$ for the right one.

ones. Rewriting matrix $M$ as

$$
M=\left(\begin{array}{cccc}
T & R_{S}^{m} & R_{S}^{\ell} & R_{P}^{m} \\
0 & * & * & *
\end{array}\right) \text { with } T=\left(\begin{array}{cc}
M_{S} & M_{S P} \\
M_{P S} & M_{P}
\end{array}\right)
$$
with $R_{U}^{v}=\left(\begin{array}{c}M_{S A_{U}^{v}} \\ M_{P A_{U}^{v}}\end{array}\right)$ with $U \in\{S, P\}$ and $v \in\{m, \ell\}$,

then starting from an initial distribution $\alpha=\left(\begin{array}{llll}\alpha_{T} & 0 & 0 & 0\end{array}\right)$, with $\alpha_{T}=\left(\alpha_{S} \alpha_{P}\right)$ then the probability $p\left(A_{S}^{\ell}\right)$ for Markov chain $X$ to be absorbed in states $A_{S}^{\ell}$ is

$$
p\left(A_{S}^{\ell}\right)=\alpha_{T}(I-T)^{-1} R_{S}^{\ell} \mathbb{1} .
$$

A similar computation gives the probabilities $p\left(A_{S}^{m}\right)$ and $p\left(A_{P}^{m}\right)$ to be respectively absorbed in states $A_{S}^{m}$ and $A_{P}^{m}$.

Figure 4 shows these different probabilities of absorption for protocol $_{1}$ with the initial distribution $\alpha=\delta$ (on the left graph) and $\alpha=\beta$ (on the right graph). Clearly in absence of adversarial behavior $(\mu=0)$, the cluster remains safe until it splits or merge, and both operations are equiprobable. Actually, we see that $p\left(A_{S}^{m}\right)=0.57$ and $p\left(A_{S}^{\ell}\right)=0.43$. This comes from the fact that the initial size $s_{0}$ of the spare set is equal to $\lfloor\Delta / 2\rfloor=\lfloor 7 / 2\rfloor=3$ (cf. Section VII-A), and thus the probability to reach a merge safe state equals $1-3 / 7 \simeq 0.57$, and thus a split safe state equals 0.43 . Thus after some initial period of growth, the topology of the overlay is stable. Let us now observe the influence of the adversary on the probabilities of absorption. For a given $\mu$, the probability for a safe cluster to split increases with larger values of $d$. This confirms the results described above: the population of the cluster increases as malicious peers trigger less leave operations than join operations. However, despite the fact that malicious peers stay longer in the cluster, for $\alpha=\delta$, the probability for the cluster to merge in a polluted state is very small (strictly less than $8 \%$ ) even for very large values of both $\mu$ (e.g., $\mu=30 \%)$ and $d$ (e.g., $d=90 \%$ ). These results are of utmost importance as they show that it is very improbable that polluted clusters manage to contaminate the other clusters of the system. As a consequence this fault-containment feature makes unlikely the probability for a cluster to start in a state in which the population of malicious peers is non negligible, that is from the initial distribution $\beta$. This is confirmed in the next Section.

\section{Modeling the AdVERsarial Strategy IN THE OVERLAY NETWORK}

We now analyze the impact of the adversary on the whole overlay, and in particular the long run proportion of polluted clusters. We consider an overlay populated with $n$ clusters $\mathcal{D}_{1}, \ldots, \mathcal{D}_{n}$, and subject to join and leave events. Each cluster $\mathcal{D}_{i}$ implements the same protocol protocol . $_{\text {. }}$ We assume that join and leave events are uniformly distributed throughout the overlay. Specifically, when a join or leave event occurs in the overlay it affects cluster $\mathcal{D}_{i}$ with probability $p_{i}=1 / n$. Thus we consider, for $n \geq 1, n$ Markov chains $X^{(1)}, \ldots, X^{(n)}$ identical to $X$, i.e. with the same state space $\Omega$, the same transition probability matrix $M$ and the same initial probability distribution $\alpha$. However these chains are not independent since, at each instant, only one Markov chain is allowed to make a transition, this Markov chain being chosen with probability $1 / n$. We denote by $N_{S}^{(n)}(m)$ and $N_{P}^{(n)}(m)$ the respective number of safe and polluted clusters just after the $m^{\text {th }}$ join or leave event, i.e. the respective number of Markov chains that are in set $S$ and in set $P$ at instant $m$. More formally, these random variables are defined, for $m \geq 0$, by

$N_{S}^{(n)}(m)=\sum_{h=1}^{n} 1_{\left\{X_{m}^{(h)} \in S\right\}}$ and $N_{P}^{(n)}(m)=\sum_{h=1}^{n} 1_{\left\{X_{m}^{(h)} \in P\right\}}$. 
It has been proved in [25] that the transient state probabilities of each Markov chain $X^{(h)}, h=1, \ldots, n$ at instant $m \geq 0$ is given by the following theorem.

Theorem 1: For every $h=1, \ldots, n, m \geq 0$ and $j \in \Omega$, we have

$$
\mathbb{P}\left\{X_{m}^{(h)}=j\right\}=\sum_{\ell=0}^{m}\left(\begin{array}{c}
m \\
\ell
\end{array}\right)\left(\frac{1}{n}\right)^{\ell}\left(1-\frac{1}{n}\right)^{m-\ell} \mathbb{P}\left\{X_{\ell}=j\right\}
$$

Note that this probability is independent of $h$ since, even though the Markov chains are dependent, they behave identically and each of them is chosen with probability $1 / n$.

The expectations of random variables $E\left(N_{S}^{(n)}(m)\right)$ and $E\left(N_{P}^{(n)}(m)\right)$ are then obtained in the following theorem. We denote by $\mathbb{1}_{S}$ (resp. $\mathbb{1}_{P}$ ) the column vector of dimension $|S \cup P|$ which $i$ th entry is equal to 1 (resp. 0) if $i \in S$ and 0 (resp. 1) if $i \in P$.

Theorem 2: For every $n \geq 1$ and $m \geq 0$, we have

$$
\begin{aligned}
\frac{E\left(N_{S}^{(n)}(m)\right)}{n} & =\alpha\left(\frac{1}{n} T+\left(1-\frac{1}{n}\right) I\right)^{m} \mathbb{1}_{S} . \\
\frac{E\left(N_{P}^{(n)}(m)\right)}{n} & =\alpha\left(\frac{1}{n} T+\left(1-\frac{1}{n}\right) I\right)^{m} \mathbb{1}_{P} .
\end{aligned}
$$

Proof: From the definition of $N_{S}^{(n)}(m)$ and using Theorem 1, we have

$$
\begin{aligned}
E\left(N_{S}^{(n)}(m)\right) & =\sum_{h=1}^{n} \mathbb{P}\left\{X_{m}^{(h)} \in S\right\}=\sum_{h=1}^{n} \sum_{j \in S} \mathbb{P}\left\{X_{m}^{(h)}=j\right\} \\
= & n \sum_{\ell=0}^{m}\left(\begin{array}{c}
m \\
\ell
\end{array}\right)\left(\frac{1}{n}\right)^{\ell}\left(1-\frac{1}{n}\right)^{m-\ell} \mathbb{P}\left\{X_{\ell} \in S\right\}
\end{aligned}
$$

It is well-known that the transient state probabilities of generic Markov chain $X$ are given by

$$
\mathbb{P}\left\{X_{\ell} \in S\right\}=\alpha T^{\ell} \mathbb{1}_{S}
$$

We thus get

$$
\begin{aligned}
E\left(N_{S}^{(n)}(m)\right) & =n \sum_{\ell=0}^{m}\left(\begin{array}{c}
m \\
\ell
\end{array}\right)\left(\frac{1}{n}\right)^{\ell}\left(1-\frac{1}{n}\right)^{m-\ell} \alpha T^{\ell} \mathbb{1}_{S} \\
& =n \alpha\left(\frac{1}{n} T+\left(1-\frac{1}{n}\right) I\right)^{m} \mathbb{1}_{S} .
\end{aligned}
$$

Expectation $E\left(N_{P}^{(n)}(m)\right)$ is obtained using the same lines.

The states of $S \cup P$ being transient, matrix $T$ is substochastic and so is matrix $T / n+(1-1 / n) I$, for every $n \geq 1$. We then have, for every $n \geq 1$,

$$
\lim _{m \rightarrow \infty} \frac{E\left(N_{S}^{(n)}(m)\right)}{n}=\lim _{m \rightarrow \infty} \frac{E\left(N_{P}^{(n)}(m)\right)}{n}=0 .
$$

Figure 5 depicts the expected proportion of safe (left figure) and polluted (right figure) clusters after the $m^{\text {th }}$ transition, i.e., that is the $m^{t h}$ join or leave operation, for realistic values of $n$ and $d$. The main observation is that the expected proportion of polluted (right figure) clusters is very low even for large values of $d$ (less that $2.2 \%$ ). Let us then observe that the expected proportion of safe clusters is almost independent of $d$ value. The same remark holds for the expected proportion of polluted clusters (even though because of the different y-axis scale used for this figure the phenomenon is not visually straightforward). This independence can be explained by the fact that the real churn dominates the induced churn (represented by parameter $d$ ).

\section{CONCLUSION}

The main lessons learnt from this study is that $(i)$ shuffling a single peer at a time ( protocol $_{1}$ ) performs better than shuffling several peers (protocol $_{k}$ with $k>1$ ). This is an interesting property because when $k=1$, the implementation is reduced to a single Byzantine tolerant agreement algorithm run amongst spare members, compared to two such runs for $k>1$ (an additional one is needed in the core set). (ii) By choosing an adequate value of $L$, i.e., the length of the lifetime of peers incarnation, it is possible to noticeably reduce the propagation of attacks in the whole system, and remarkably decrease the overhead of the induced churn at cluster level. This is another interesting result as it demonstrates that there is no need to keep the system in an hyper-activity to be resilient against targeted attacks. Pushing peers smoothly but to unpredictable regions of the system is sufficient.

\section{REFERENCES}

[1] N. Naoumov and K. W. Ross, "Exploiting p2p systems for ddos attacks," in Proceedings of the International Conference on Scalable Information Systems (Infoscale), 2006.

[2] A. Fiat, J. Saia, and M. Young, "Making chord robust to byzantine attacks," in Proceedings of the Annual European Symposium on Algorithms (AESA), 2005.

[3] I. Baumgart and S. Mies, "S/kademlia: A practicable approach towards secure key-based routing," in Proceedings of the International Conference on Parallel and Distributed Systems (ICPADS), 2007.

[4] E. Anceaume, F. Brasileiro, R. Ludinard, and A. Ravoaja, "PeerCube: an hypercube-based p2p overlay robust against collusion and churn," in Proceedings of the International Conference on Self-Adaptive and Self-Organizing Systems (SASO), 2008.

[5] B. Awerbuch and C. Scheideler, "Towards scalable and robust overay networks," in Proceedings of the International Workshop on Peer-to-Peer Systems (IPTPS), 2007.

[6] P. B. Godfrey, S. Shenker, and I. Stoica, "Minimizing churn in distributed systems," in Proceedings of the ACM SIGCOMM, 2006. 


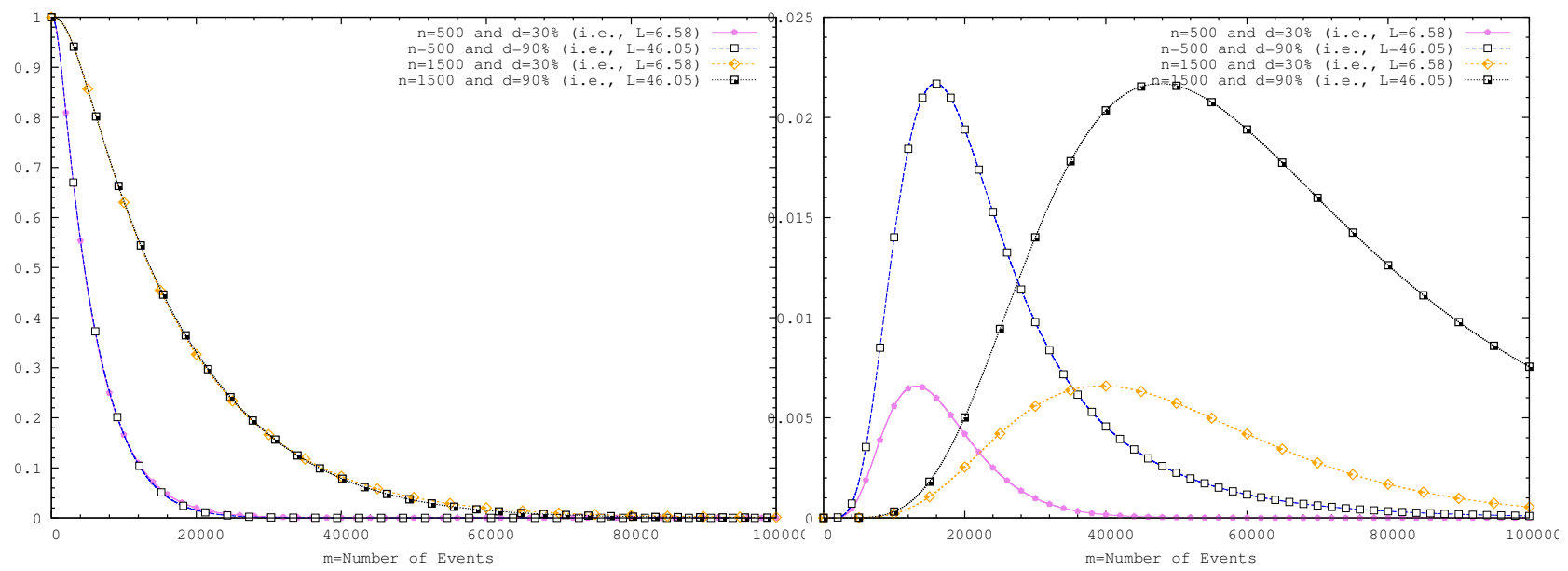

Figure 5: $\frac{E\left(N_{S}^{(n)}(m)\right)}{n}$ and $\frac{E\left(N_{P}^{(n)}(m)\right)}{n}$ as a function of $m$ for different values of $n$ and $d$.

[7] E. Anceaume, F. Brasileiro, R. Ludinard, B. Sericola, and F. Tronel, "Analytical study of adversarial strategies in clusterbased overlays," in Proceedings of the 2nd International Workshop on Reliability, Availability, and Security (WRAS), 2009.

[8] M. Castro, P. Druschel, A. Ganesh, A. Rowstron, and D. S. Wallach, "Secure routing for structured peer-to-peer overlay networks," in Proceedings of the Symposium on Operating Systems Design and Implementation (OSDI), 2002.

[9] S. Ratnasamy, P. Francis, M. Handley, R. Karp, and S. Shenker, "A scalable content-addressable network," in Proceedings of the ACM SIGCOMM, 2001.

[10] I. Stoica, D. Liben-Nowell, R. Morris, D. Karger, F. Dabek, M. F. Kaashoek, and H. Balakrishnan, "Chord: A scalable peer-to-peer lookup service for internet applications," in Proceedings of the ACM SIGCOMM, 2001.

[11] P. Druschel and A. Rowstron, "Past: A large-scale, persistent peer-to-peer storage utility," in Proceedings of the Workshop on Hot Topics in Operating Systems (HotOS), 2001.

[12] A. Singh, T. Ngan, P. Drushel, and D. Wallach, "Eclipse attacks on overlay networks: Threats and defenses," in Proceedings of the Conference on Computer Communications (INFOCOM), 2006.

[13] E. Sit and R. Morris, "Security considerations for peer-to-peer distributed hash tables," in Proceedings of the International Workshop on Peer-to-Peer Systems (IPTPS), 2002.

[14] K. Hildrum and J. Kubiatowicz, "Asymptotically efficient approaches to fault-tolerance in peer-to-peer networks," in Proceedings of theInternational Symposium on Distributed Computing (DISC), 2003.

[15] T. Condie, V. Kacholia, S. Sankararaman, J. M. Hellerstein, and P. Maniatis, "Induced churn as shelter from routing-table poisoning," in Procs of the 13th thirteenth Annual Symposium on Network and Distributed System Security (NDSS'06), 2006.
[16] C. G. Plaxton, R. Rajaraman, and A. W. Richa, "Accessing nearby copies of replicated objects in a distributed environment," in Proceedings of the 9th Annual ACM Symposium on Parallel Algorithms and Architectures (SPAA), 1997.

[17] P. Maymounkov and D. Mazieres, "Kademlia: A peer-to-peer information system based on the XOR metric," in Proceedings for the International Workshop on Peer-to-Peer Systems (IPTPS), 2002.

[18] T. Locher, S. Schmid, and R. Wattenhofer, "eQuus: A provably robust and locality-aware peer-to-peer system," in Proceedings of the International Conference on Peer-to-Peer Computing $(P 2 P), 2006$.

[19] J. Douceur, "The sybil attack," in Proceedings of the International Workshop on Peer-to-Peer Systems (IPTPS), 2002.

[20] R. Housley, W. Ford, W. Polk, and D. Solo, "Internet x.509 public key infrastructure certificate and crl profile," 1999.

[21] L. Lamport, R. Shostak, and M. Pease, "The byzantine generals problem," ACM Transactions on Programming Languages and Systems, vol. 4, 1982.

[22] E. Anceaume, R. Ludinard, B. Sericola, and F. Tronel, "Performance analysis of large scale peer-to-peer overlays using markov chains," IRISA, http://hal.inria.fr/inria-00546039/en/, Tech. Rep. 1963, 2010.

[23] B. Sericola, "Closed form solution for the distribution of the total time spent in a subset of states of a Markov process during a finite observation period," Journal of Applied Probability, vol. 27, no. 3, pp. 713-719, 1990.

[24] B. Sericola and G. Rubino, "Sojourn times in Markov processes," Journal of Applied Probability, vol. 26, no. 4, pp. 744-756, 1989.

[25] E. Anceaume, F. Castella, R. Ludinard, and B. Sericola, "Markov chains competing for transitions: Application to large scale distributed systems," INRIA, http://hal.inria.fr/inria-00485667/en/, Tech. Rep., 2011. 